\title{
Scattering of slow electrons by polar molecules: Application of effective-range potential theory to $\mathrm{HCl}$
}

\author{
Wim Vanroose, ${ }^{*}$ C. W. McCurdy, ${ }^{\dagger}$ and T. N. Rescigno ${ }^{\ddagger}$ \\ Computing Sciences, Lawrence Berkeley National Laboratory, 1 Cyclotron Road, Berkeley, California 94720, USA
}

(Received 19 June 2003; published 21 November 2003)

\begin{abstract}
We present a nonempirical potential model for studying threshold vibrational excitation of polar molecules by electron impact. This work builds on the zero-range potential virtual state model of Gauyacq and Herzenberg [J. P. Gauyacq and A. Herzenberg, Phys. Rev. A 25, 2959 (1982)], using known analytic properties of the $S$ matrix for a dipole potential to predict the analytic continuation of the negative ion potential curve into the continuum. We derive an equation that determines the nuclear dynamics which can be solved without the need for an expansion in target vibrational states. The model is applied to $e^{-}-\mathrm{HCl}$ and is found to capture the essential features of the observed excitation cross sections, including both the threshold peaks as well as oscillatory structures at energies above threshold.
\end{abstract}

DOI: 10.1103/PhysRevA.68.052713

PACS number(s): $34.80 . \mathrm{Gs}$

\section{INTRODUCTION}

Vibrational excitation of the hydrogen halides by lowenergy electron impact has continued to attract the attention of experimentalists and theorists for many years. Of particular interest are the pronounced threshold peaks in the vibrationally inelastic cross sections which were first observed by Rohr and Linder some 25 years ago [1]. These early observations prompted considerable debate about mechanisms that might be responsible for the observed structures and a variety of different explanations were put forth.

Two models were initially proposed to explain the observed threshold peaks. Dubé and Herzenberg [2] and Gauyacq and Herzenberg [3] argued that shape resonances could not be involved since the observed angular distributions at threshold were isotropic, suggesting a strong $s$-wave component (the electron collision energies are less than $1 \mathrm{eV}$ ), which in turn argued against a resonant trapping mechanism. The zero-range potential model they developed assumed that the problem involves only $s$-wave scattering. A virtual state mechanism was proposed to account for the enhancement of the wave function of a slow exiting electron. When vibrational motion was introduced into the fixed-nuclei picture, nuclear-excited Feshbach resonances appeared below the vibrational thresholds and, in their view, were responsible for the observed structures.

A different model, based on a nonlocal projection operator theory, was proposed by Domcke and Cederbaum [4]. Their treatment rests on the picture of a discrete state interacting with a continuum and makes use of a projection operator resonance formalism [5] that provides a formally exact description of the nuclear motion with nonlocal complex potentials. In their treatment, the threshold behavior of the cross sections is a consequence of the strong coupling between a discrete resonance and a background dipole con-

\footnotetext{
*Electronic address: wivanroose@lbl.gov

†Electronic address: cwmccurdy@lbl.gov

‡Electronic address: tnrescigno@1bl.gov
}

tinuum that mixes $s$ and $p$ waves. The zero-range potential model and the nonlocal projection operator theory were both parametrized to give a successful accounting of the observed threshold peaks.

The early models were eventually followed by a series of $a b$ initio calculations, most notably nonadiabatic $R$ matrix calculations [6] that included explicit coupling between electronic and nuclear degrees of freedom, for $e^{-}$-HF [7], $e^{-}-\mathrm{HCl}[8]$, and $e^{-}-\mathrm{HBr}$ [9] scattering. These calculations were successful in accounting for the qualitative behavior of the observed cross sections. Moreover, the $R$-matrix results, and subsequent theoretical analyses $[10,11]$ of their associated analytic structure, gave a picture of the dynamics that was consistent with a nuclear-excited Feshbach resonance mechanism.

What all of these approaches have shown is that the simple picture of a resonance, which lies close to the real energy axis for compressed nuclear geometries and adiabatically evolves into an electronically bound negative ion as the internuclear distance increases, is drastically modified in the case of a polar molecule. Fandreyer and Burke's [11] analysis of the $e^{-}-\mathrm{HBr} S$ matrix revealed that there was a shape resonance, but the trajectory it traced when the internuclear distance was varied was disconnected from that of bound $\mathrm{HBr}^{-}$. As the molecule is stretched from its equilibrium value, the resonance is turned away from the real axis, moves off into the lower half of the complex momentum $(k)$ plane, and never becomes a bound state.

A bound anion state does appear for larger internuclear distances, but its behavior as the internuclear distance is decreased is profoundly affected by the underlying electrondipole interaction [12]. A polar molecular anion cannot have a true "virtual state," that is, a state with a purely imaginary $k$ value in the lower half plane, close to the real axis. If we track the pole position of a bound diatomic anion as the internuclear separation is decreased, then for some critical distance $R_{o}$ the binding energy will go to zero. If the distance is further decreased, then the pole passes through $k=0$ into the lower half of the complex momentum plane. For a nonpolar system, this trajectory would be one that moves 
straight down the negative imaginary $k$ axis. But for a polar system, there is a branch cut along this axis and the pole splits into mirror-image pairs on higher Riemann sheets as it passes through the origin [13]. The virtual state trajectory thus possesses a discontinuous slope or "kink" as it passes through the origin; the angles at which the mirror-image virtual state poles emerge from the origin are uniquely determined by the value of the dipole moment at $R_{c}[12,13]$. The fact that the bound-state and resonance trajectories do not connect is a direct consequence of the dipole field that strongly mixes the $s$ - and $p$-wave continua and is an example of generalized level repulsion in the continuum [14-17]. This analytic structure was also predicted by Domcke [18] and confirmed by Fandreyer and Burke's calculations [11].

The nonlocal resonance model has been extensively refined over the past two decades and has been very successful in reproducing details of the experimentally measured $e^{-}$-HX cross sections $[19,20]$. In the case of $\mathrm{HCl}[19]$, for example, it reproduces both the threshold peaks and the higher-energy structure caused by an outer well in the bound portion of the $\mathrm{HCl}^{-}$potential-energy curve. This level of agreement would seem to indicate that the low-energy collision dynamics between electrons and weakly polar diatomic molecules is completely understood. So what motivation, one may ask, is there for revisiting this problem?

Our primary objective here is the formulation of an $a b$ initio model which captures the essential features of the observed cross sections at low energies, does not require an elaborate parametrization in its execution and is applicable to more complicated systems. This in turn has prompted us to reexamine the zero-range potential model. Like Gauyacq and Herzenberg [3], we use effective-range theory as the starting point for deriving an equation that determines the nuclear dynamics. What we end with is a model which, structurally at least, resembles the local complex potential or "Boomerang" model [21] which has proven to be a very useful tool in describing resonant vibrational excitation, i.e., a complex, inhomogeneous wave equation which determines the lowenergy collision cross sections. Furthermore, the equation can be solved without resorting to an expansion in target vibrational states. Another element in our formulation is the use of a dipole coupled partial-wave model to predict the analytic continuation of the negative-ion potential curve into the continuum, which allows one to build a nonempirical model whose only input is the potential curve of the anion in the region where it is bound, the potential curve of the target, and its $R$-dependent dipole moment.

Like the boomerang model, which has recently been extended to look at resonant nuclear motion in several dimensions [22,23], our effective-range model can be extended to polyatomics, with only modest computational requirements, provided a suitable anion potential surface can be constructed. Interesting threshold structures have, in fact, been observed in the vibrational excitation cross sections of polyatomic targets, such as $\mathrm{CO}_{2}$ [24] and $\mathrm{CS}_{2}$ [25], which are nonpolar in their equilibrium geometry, but acquire a transient dipole moment upon bending. While our initial applications here are focused on $e^{-}-\mathrm{HCl}$, the application of this model to polyatomic targets has provided additional motiva- tion for the present development.

The outline of this paper is as follows. The theory is presented in the following two sections. In Sec. II, we discuss the procedure we use to construct the adiabatic potential curve for the negative ion and its analytic continuation into the complex momentum plane. The derivation of the effective-range model is then outlined in Sec. III. Our results for $e^{-}-\mathrm{HCl}$ vibrational excitation are presented in Sec. IV. Section V contains some concluding remarks.

\section{POTENTIAL CURVE OF THE NEGATIVE ION}

The principal assumption of the effective-range model, for very low-energy electron scattering by polar molecules, is that the target provides the electron with a potential well that is on the verge of binding an extra electron. Thus small displacements of the nuclei about their equilibrium position can cause this bound state to appear or vanish. To describe this dynamics, we must be able to construct an adiabatic potential curve for the molecular anion. This is not a problem for nuclear geometries where the anion is electronically bound: one can perform $a b$ initio structure calculations for the bound portion of the potential curve. For the unbound portion of the curve, we rely on an analysis of the long-range part of the electron-molecule interaction, which is dominated by the dipole field.

The binding properties of a fixed dipole potential are well known and have been studied by a number of authors. Here, we follow the treatment of Lévy-Leblond [26]. The Schrödinger equation for an electron in a dipole field is given by

$$
\left(-\frac{1}{2} \Delta+e \frac{\mathbf{D}(R) \cdot \mathbf{r}}{r^{3}}-E\right) \psi(r, \theta, \phi ; R)=0,
$$

where $\mathbf{D}$ is the dipole moment and depends on the internuclear separation $R$. Equation (1) is separable in spherical polar coordinates. Choosing the $z$ axis to coincide with the dipole moment $\mathbf{D}$ and writing

$$
\psi(r, \theta, \phi ; R)=\frac{1}{r} P(r) \Theta(\theta) e^{i m \phi}
$$

gives the following equations for the radial and angular parts of the wave function:

$$
\left(-\frac{d^{2}}{d r^{2}}+\frac{l_{n}\left(l_{n}+1\right)}{r^{2}}-2 E\right) P(r)=0
$$

and

$$
\begin{gathered}
{\left[\frac{1}{\sin \theta} \frac{d}{d \theta}\left(\sin \theta \frac{d}{d \theta}\right)-\frac{m^{2}}{\sin ^{2} \theta}-2 D \cos \theta\right.} \\
\left.+l_{n}\left(l_{n}+1\right)\right] \Theta_{n}(\theta)=0,
\end{gathered}
$$

where $l_{n}\left(l_{n}+1\right)$ is a separation constant and $m$ is an integer. The separation constant is written in the form of an effective 
angular momentum $l_{n}$ and is obtained by solving for the eigenvalues of the angular equation, Eq. (4). We need only consider the lowest eigenvalue $l_{0}$ for the $m=0$ case since this is the only angular mode that gives rise to an attractive centrifugal potential in Eq. (3) [2]. Lévy-Leblond [26] considered this problem and derived a power series for the solution:

$$
\begin{aligned}
l_{0}(R)\left[l_{0}(R)+1\right]= & -\frac{2 D(R)^{2}}{3}+\frac{11}{30}\left(\frac{2 D(R)^{2}}{3}\right)^{2} \\
& -\frac{133}{450}\left(\frac{2 D(R)^{2}}{3}\right)^{3}+\cdots
\end{aligned}
$$

For a subcritical dipole moment $\left(D<0.639 e a_{o}\right)$, the centrifugal term $l_{0}$ will be a nonintegral negative number between $-\frac{1}{2}$ and zero. We will henceforth drop the subscript 0 on $l$ with the understanding that it refers to the solution of Eq. (5).

A nonintegral $l$ value in the radial equation leads to a multivalued Jost function $\mathcal{F}_{l}(K)[13,27]$. Indeed, close to the origin, the Jost function for continuous angular momentum [12] reduces to a series that has terms that have noninteger exponents,

$$
\begin{aligned}
\mathcal{F}_{l}(K ; R)= & a_{0}(R)+a_{1}(R) K^{2}+\cdots+b_{1}(R) K^{2 l+1} \\
& +b_{2}(R) K^{2 l+3}+\cdots,
\end{aligned}
$$

where $K$ is the momentum of the electronic state relative to the threshold. A zero of the Jost function $\mathcal{F}_{l}(K ; R)$ corresponds to a pole of the $S$ matrix. For a bound state, the zero lies on the positive imaginary $K$ axis. Its behavior near threshold $(K=0)$ depends critically on $l$, which in turn depends on the dipole moment through Eq. (5). Since $l$ is negative for the case of a subcritical dipole, the $K^{2 l+1}$ term dominates the $K^{2}$ term in Eq. (6), so close to the threshold, the most important terms of the Jost function are

$$
\mathcal{F}_{l}(K ; R) \simeq a_{0}(R)+b_{1}(R) K^{2 l+1} .
$$

Let $R_{o}$ denote the nuclear coordinate at which the electron becomes unbound, i.e., $K\left(R_{o}\right)=0$, and expand the coefficients $a_{0}(R)$ and $b_{1}(R)$ about that point. We find that the condition for a zero of the Jost function near threshold is

$$
0=a_{0}\left(R_{o}\right)^{\prime}\left(R-R_{o}\right)+b_{1}\left(R_{o}\right) K^{2 l+1}
$$

or

$$
K(R)=i \beta\left(R-R_{o}\right)^{1 /[2 l(R)+1]},
$$

where we have defined

$$
i \beta \equiv\left[-a_{0}\left(R_{o}\right)^{\prime} / b_{1}\left(R_{o}\right)\right]^{1 /[2 l(R)+1]} .
$$

We can now contrast the case of pure $s$-wave scattering from a nonpolar target with the polar case. When $D$ vanishes, $l$ is zero and we get

$$
K(R) \propto i\left(R-R_{o}\right),
$$

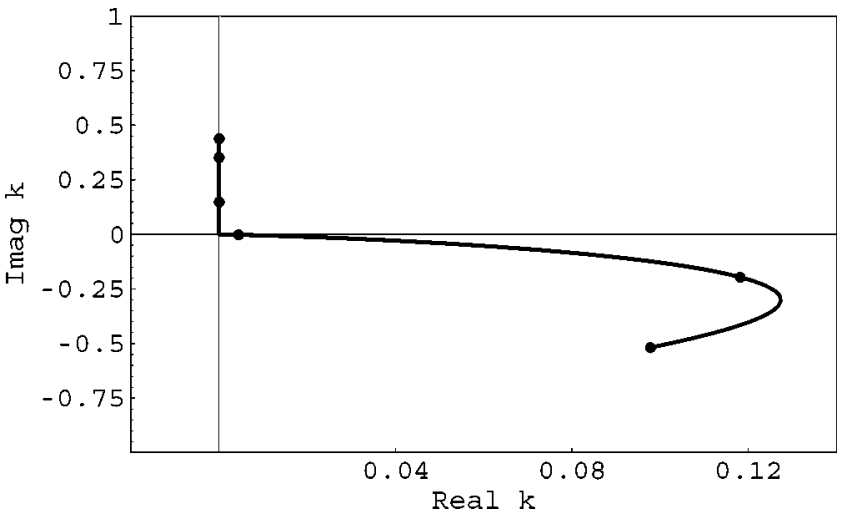

FIG. 1. Trajectory of a pole of the $S$ matrix in the complex $K$ plane for the dipole potential problem discussed in text. We used a dipole moment function appropriate for $\mathrm{HCl}$. The circles correspond to internuclear distance values of 1.25, $1.92276,2.5,3.07724,3.75$ and 4.42276 bohrs.

that is, the pole moves linearly along the imaginary $k$ axis as $R$ passes through $R_{o}$. For a weakly polar target, $l(R) \simeq$ $-2 D^{2} / 3$ and we have, for small $D$,

$$
K(R) \propto i\left(R-R_{o}\right)^{1 /\left[1-\left(4 D^{2} / 3\right)\right]} \approx i\left(R-R_{o}\right)^{1+\left(4 D^{2} / 3\right)} .
$$

There is now a branch point at $R=R_{o}$; if $D$ is irrational, there are infinitely many branches. We will put the branch line along the negative imaginary axis.

For $R>R_{o}, k$ is positive imaginary, corresponding to a bound state. As $R$ decreases through $R_{o}, k$ becomes complex. We will consider its trajectory on what Herzenberg [13] calls "the first counterclockwise sheet," that is, measuring the phase of $k$ counterclockwise from the positive real axis. From Eq. (12), we get

$$
K(R) \propto\left(R_{o}-R\right)^{1+\left(4 D^{2} / 3\right)} e^{i \pi\left[(3 / 2)+\left(4 D^{2} / 3\right)\right]}, \quad R<R_{o} .
$$

The phase of $k$ is greater than $3 \pi / 2$, i.e., it is in the fourth quadrant of the second Riemann sheet, a direct consequence of the attractive nature of the effective potential. There is a mirror-image pole in the third quadrant with a phase angle of $-\pi\left(1 / 2+4 D^{2} / 3\right)$, as well as mirror-image pairs on higher Riemann sheets. These we ignore.

We show the trajectory of a bound state in Fig. 1 for a case where the dipole strength decreases monotonically with $R$. The decreasing dipole strength causes the trajectory to bend back toward the imaginary $k$ axis after it passes through the origin.

With the effective electron momentum defined by Eq. (9), we construct the negative-ion potential curve as

$$
V_{\text {ion }}(R)=V_{\text {neutral }}(R)+\frac{1}{2} K(R)^{2} .
$$

By construction, $V_{\text {ion }}$ crosses $V_{\text {neutral }}$ at $R_{o}$ and acquires a negative imaginary part for $R<R_{o}$. For $R>R_{o}, V_{i o n}$ is real and can be calculated by ab initio techniques. For our purposes, it is presumed to be known. The single parameter $\beta$ in Eq. (9) is chosen so that we get a smooth connection between the inner and outer portions of the ion curve. 
The behavior of $V_{i o n}$ differs markedly from what is seen in the case of a shape resonance. In the extreme case of a pure virtual state that can exist in the absence of a dipole moment, the trajectory of $K(R)$ would follow the simple parametrization $K(R)=i\left(R-R_{o}\right)$, changing sign as $R$ passes through $R_{o}$. But $\frac{1}{2} K(R)^{2}=-\frac{1}{2}\left(R-R_{0}\right)^{2}$ is always real and negative, independent of the sign of $\left(R-R_{0}\right)$. This means that for a pure virtual state, the $V_{\text {ion }}$ curve is real and lies below $V_{\text {neutral }}$ except at the point $R_{o}$ where they touch. The introduction of a weak dipole moment does not dramatically alter this picture: $K(R)$ will move off the imaginary axis as it passes through the origin and $V_{\text {ion }}$ will acquire a negative imaginary component. But for a weak dipole moment, the real part of $V_{\text {ion }}$ will generally fall below the $V_{\text {neutral }}$ potential curve.

We are now in a position to derive the nuclear wave equation of our dynamical model.

\section{NUCLEAR DYNAMICS IN THE EFFECTIVE-RANGE MODEL}

The basic idea behind the effective-range model is that for very low-energy scattering the wave function which describes the scattered electron is independent of energy inside some radius $r_{o}$ [28]. Inside $r_{o}$, the potential is strong and the electron follows the nuclei adiabatically. The logarithmic derivative of wave function at $r=r_{o}$,

$$
f(R)=\left(\frac{1}{\psi(r ; R)} \frac{\partial \psi(r ; R)}{\partial r}\right)_{r=r_{o}},
$$

is introduced to avoid calculations in the inner region. The log-derivative depends on the internuclear geometry, but is assumed to be independent of the collision energy, on the assumption that the latter is small compared to the internal potential.

Using $S$-matrix boundary conditions, the wave function in the outer region $r>r_{o}$ is replaced by the asymptotic form corresponding to the lowest angular mode of the fixed dipole problem:

$$
\psi(r ; R)=h_{l}^{-}\left(k_{0} r\right) \chi_{0}(R)+\sum_{n} A_{n} h_{l}^{+}\left(k_{n} r\right) \chi_{n}(R),
$$

where $\chi_{n}(R)$ is a target vibrational function with energy $E_{n}$, $k_{n}=\sqrt{2\left(E-E_{n}\right)}$ is the electron channel momentum and $h^{+(-)}$is an outgoing (incoming) Hankel function. Note that only the $A_{n}$ associated with real (open) $k_{n}$ contribute to the cross sections. These in turn are determined by matching the $\log$-derivative of $\psi$ at $r_{o}$. The log-derivative $f(R)$ is generally treated as a semiempirical parameter, but with the information of the preceding section, it can be determined.

We assume that the wave function in the inner region can be equated with the adiabatic Siegert state associated with $V_{i o n}$, which is a purely outgoing wave at large $r$,

$$
\lim _{r \rightarrow \infty} \psi(r ; R) \sim \exp [i K(R) r-l(R) \pi / 2],
$$

where $K(R)$ is defined by the relative spacing between the neutral and the anion surface [Eq. (14)]:

$$
K(R)=\sqrt{2\left[V_{\text {ion }}(R)-V_{\text {neutral }}(R)\right]} .
$$

It follows that $f(R)=i K(R)$.

Matching the logarithmic derivatives of Eqs. (17) and (16) at $r=r_{o}$ leads to

$$
i K(R)=\frac{k_{0} h_{l}^{-}\left(k_{0} r_{o}\right)^{\prime} \chi_{0}(R)+\sum_{n} k_{n} A_{n} h_{l}^{+}\left(k_{n} r_{o}\right)^{\prime} \chi_{n}(R)}{h_{l}^{-}\left(k_{0} r_{o}\right) \chi_{0}(R)+\sum_{n} A_{n} h_{l}^{+}\left(k_{n} r_{o}\right) \chi_{n}(R)} .
$$

We further reorganize the matching equation using the fact that $\chi_{n}$ is a vibrational state of the neutral Hamiltonian $H_{\text {neutral }}$,

$$
k_{n} \chi_{n}(R)=\sqrt{2\left(E-E_{n}\right)} \phi_{n}(R)=\sqrt{2\left[E-H_{\text {neutral }}(R)\right]} \chi_{n}(R),
$$

and replace each appearance of $k_{n}$ with the operator $\sqrt{2\left[E-H_{\text {neutral }}(R)\right]}$. The matching equation now becomes

$$
\begin{aligned}
i K(R)\left[h_{l}^{-}\left(k_{0} r_{o}\right) \chi_{0}(R)+\sum_{n} A_{n} h_{l}^{+}\left(k_{n} r_{o}\right) \chi_{n}(R)\right] \\
=\sqrt{2\left(E-H_{\text {neutral }}\right)}\left[h_{l}^{-}\left(k_{0} r_{o}\right)^{\prime} \chi_{0}(R)\right. \\
\left.\quad+\sum_{n} A_{n} h_{l}^{+}\left(k_{n} r_{o}\right)^{\prime} \chi_{n}(R)\right] .
\end{aligned}
$$

Equation (21) can be further simplified if we assume that $r_{o}$ can be chosen large enough so that the Hankel functions can be replaced by their asymptotic forms, $h_{l}^{+/-}(k r) \sim \exp$ $(+/-i k r-l \pi / 2)$. With this assumption Eq. (21), after rearrangement, becomes

$$
\begin{aligned}
{[-} & \left.K(R)+\sqrt{2\left(E-H_{\text {neutral }}\right)}\right] \sum_{n} A_{n} e^{i\left(k_{n}+k_{0}\right) r_{o}} \chi_{n}(R) \\
& =\left[K(R)+k_{0}\right] \chi_{0}(R) .
\end{aligned}
$$

Note that the factors $e^{i\left(k_{0}+k_{n}\right) r_{o}}$ can be incorporated into a redefinition of the coefficients $A_{n}$ without changing the excitation cross sections, which are proportional to $\left|A_{n}\right|^{2}$. So the matching equation is now independent of $r_{o}$, as in the zerorange potential model [28].

Equation (22) can be converted to a set of linear equations for the unknown coefficients $A_{n}$ by multiplying from the left with $\chi_{n}$ and integrating over $R$. This is the procedure used in Refs. [2,3]. There can, however, be convergence problems with such a linear system [28]. In many cases, the negativeion curve is very different from the neutral curve and may even be dissociative, in which case the expansion in vibrational states of the neutral target may not converge. 
Equation (22) can be solved without an expansion in target vibrational states. If we define the nuclear wave function $\Psi(R)$ as

$$
\Psi(R)=\sum_{n} A_{n} e^{i\left(k_{n}+k_{0}\right) r_{o}} \chi_{n}(R)
$$

we obtain the following inhomogeneous differential equation:

$$
\left[-K(R)+\sqrt{2\left(E-H_{\text {neutral }}\right)}\right] \Psi(R)=\left[K(R)+k_{0}\right] \chi_{0}(R) .
$$

Equation (24) can easily be solved in ways that avoid the convergence problems associated with an expansion in target vibrational states.

To solve Eq. (24), we must specify appropriate boundary conditions. For that purpose, it is useful to recast Eq. (24) into the form of a driven Schrödinger equation. If we define

$$
\Psi(R)=\left[K(R)+\sqrt{2\left(E-H_{\text {neutral }}\right)}\right] \Omega(R),
$$

we can then write Eq. (24) as

$$
\begin{aligned}
& {\left[-K(R)+\sqrt{2\left(E-H_{\text {neutral }}\right)}\right][K(R)} \\
& \left.\quad+\sqrt{2\left(E-H_{\text {neutral }}\right)}\right] \Omega(R)=\left[K(R)+k_{0}\right] \chi_{0}(R)
\end{aligned}
$$

or, using Eq. (14), as

$$
\begin{aligned}
& \left\{2\left(E-H_{\text {ion }}\right)+\left[\sqrt{2\left(E-H_{\text {neutral }}\right)}, K(R)\right]\right\} \Omega(R) \\
& \quad=\left[K(R)+k_{0}\right] \chi_{0}(R) .
\end{aligned}
$$

As $R \rightarrow \infty, K(R)$ goes to a constant and the commutator in Eq. (27) vanishes, giving

$$
\left(E-H_{\text {ion }}\right) \Omega(R)=\frac{1}{2}\left[K(R)+k_{0}\right] \chi_{0}(R), \quad R \rightarrow \infty .
$$

It is clear from Eq. (28) that $\Omega(R)$ is effectively the wave function that describes the temporary molecular state that moves asymptotically on the negative-ion curve. For total energies $E$ below the dissociative attachment threshold, $\Omega(R)$ will go to zero as $R \rightarrow \infty$, while above the dissociation threshold, the appropriate boundary condition for $\Omega(R)$ is that it behave asymptotically as a purely outgoing wave. Having solved the nuclear wave equation (NWE), Eq. (27), the excitation amplitudes are then evaluated by projecting the solution onto a target vibrational state:

$$
A_{n}=\int \chi_{n}(R) \Psi(R) d R=\int \chi_{n}(R)\left[K(R)+k_{n}\right] \Omega(R) d R .
$$

If we drop the commutator in Eq. (27) entirely, on the assumption, in the sprit of the Born-Oppenheimer (BO) approximation, that the nuclear kinetic-energy operator commutes with the logarithmic derivative defined in Eq. (15), then we need only to deal with an inhomogeneous Schrödinger equation (BO-NWE), Eq. (28), at all $R$. In the case of a polar target, however, the behavior of $K(R)$ as it passes

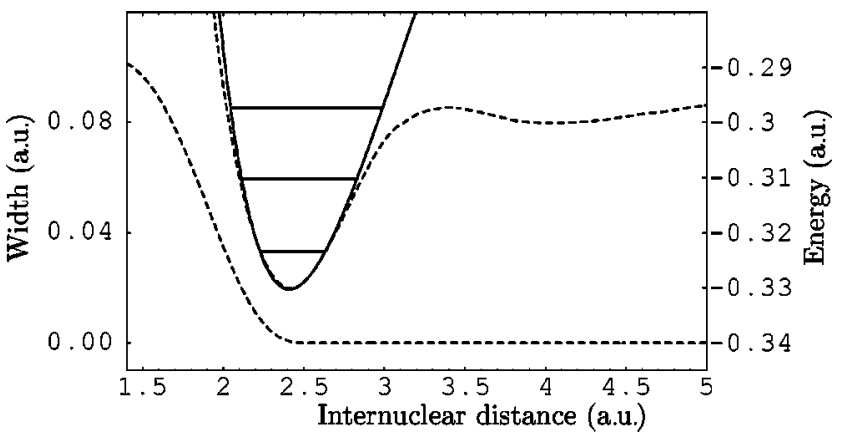

FIG. 2. Neutral and anion potential curves for $\mathrm{HCl}$. Solid curve, neutral ground state with lowest three vibrational levels indicated and dashed curves, real and imaginary parts of anion curve.

through the origin (Fig. 1) invalidates this approximation. Consequently, as we will see below, the NWE and BO-NWE can give very different results close to vibrational thresholds.

\section{APPLICATION TO HCl}

We have applied the previously outlined theory to the case of $e^{-}-\mathrm{HCl}$ vibrational excitation. The parameters required for the nuclear wave equation were obtained as follows. For the bound portion of the $\mathrm{HCl}^{-}$potential curve, we used the $a b$ initio configuration-interaction calculations of Astrand and Karlstrom [29]. Their calculations give a ${ }^{2} \Sigma \mathrm{HCl}^{-}$curve that has a shallow outer well centered at 4 bohr and crosses the neutral $\mathrm{HCl}$ ground-state curve near $2.5 \mathrm{bohr}$. For the ground-state $\mathrm{HCl}$ curve, we used a Morse potential with parameters taken from the work of Cizek, Horacek, and Domcke [30]. The analytic continuation of the $\mathrm{HCl}^{-}$curve was carried out using the dipole coupled partial-wave model outlined in Sec. II. This procedure requires the $R$-dependent dipole moment of the neutral target. This data was taken from the $a b$ initio calculations of ONeil, Rosmus, Norcross, and Werner [31]. We used a value of $2.54 \mathrm{bohr}$ for the crossing point $R_{o}$. Finally, we chose $\beta=0.4$ in Eq. (9) to smoothly connect the real and complex portions of the anion curve. The potential curves for $\mathrm{HCL}$ and $\mathrm{HCl}^{-}$we used are depicted in Fig. 2.

The ion curve is obtained by adding $\frac{1}{2} K(R)^{2}$ to the neutral $\mathrm{HCl}$ potential [Eq. (14)]. For $R>R_{o}, K(R)$ is positive imaginary; $V_{i o n}$ is purely real and lies below $V_{\text {neutral }}$, corresponding to an electronically bound state. For $R<R_{o}$, $K(R)$ lies in the fourth quadrant of the complex plane and $V_{\text {ion }}$ is complex. Since $K(R)$ stays relatively close to the negative imaginary axis, the real part of $V_{\text {ion }}$ stays below $V_{\text {neutral }}$.

To solve the NWE [Eq. (27)] and BO-NWE [Eq. (28)] equations, we used a discrete variable representation (DVR) of the operators $H_{\text {neutral }}(R)$ and $K(R)$ based on Lobatto shape functions [32]. The DVR provides diagonal representation of any local operator $\left[K(R)\right.$ and $V_{\text {neutral }}(R)$, in this case], while the matrix elements of the derivative operators needed to express the nuclear kinetic energy are given by simple analytic expressions [32]. For calculations at energies above the threshold for dissociative attachment, we need to impose outgoing-wave boundary conditions in solving the 
NWE and BO-NWE equations. This is easily accomplished within the DVR [33] by mapping the internuclear coordinate with the following exterior complex scaling transformation

$$
R \rightarrow M(R)= \begin{cases}R, & R<R_{0} \\ R_{0}+\left(R-R_{0}\right) e^{i \eta}, & R \geqslant R_{0},\end{cases}
$$

where $R_{0}$ is lies outside the interaction region. Exterior complex scaling automatically imposes the outgoing-wave boundary condition [33]. The NWE and BO-NWE are then replaced by a set of complex linear equations. For the NWE, we need a representation of the operator $\sqrt{2\left(E-H_{\text {neutral }}\right)}$. This is accomplished by diagonalizing $H_{\text {neutral }}$ in the DVR basis and expressing the operator as

$$
\sqrt{2\left(E-H_{\text {neutral }}\right)} \approx \sum_{i} u_{i}(R) \sqrt{2\left(E-E_{i}\right)} u_{i}\left(R^{\prime}\right),
$$

where $E_{i}$ and $u_{i}$ are the eigenvalues and eigenvectors of $H_{\text {neutral }}$ in the finite DVR basis. 200 DVR functions on a 20 bohr interval with $R_{0}=13$ bohrs were found to give converged results. The excitation coefficients, given by Eq. (29), were also obtained using Gauss-Lobatto quadrature with a DVR representation of the target vibrational states. The vibrational excitation cross sections are expressed in terms of the excitation coefficients by the formula

$$
\sigma_{0 n}=\frac{\pi}{k_{0}^{2}} \frac{k_{n}}{k_{0}}\left|A_{n}\right|^{2}
$$

In Fig. 3, we plot the $0 \rightarrow 1$ and $0 \rightarrow 2$ cross sections obtained from the NWE and BO-NWE models. The most significant differences between the two models are evident in the immediate vicinity of the $n=1$ and $n=2$ vibrational thresholds, where the NWE produces sharper threshold structures. The threshold regions were found to be quite sensitive to the detailed behavior of $K(R)$ in the crossing region. Unfortunately, the calculations of Astrand and Karlstrom [29], which we used in the construction of the anion curve, give only three points in the vicinity of the crossing, making it difficult to determine precise values for both $\beta$ and $R_{o}$. Small changes in $R_{o}$ alter the magnitude and shape of the threshold peaks, but this sensitivity is greatly reduced as soon as the collision energy is a few tenths of an $\mathrm{eV}$ above a vibrational threshold, where the NWE and BO-NWE models give similar results. The oscillatory structures between 0.7 and $0.8 \mathrm{eV}$ are associated with quasibound levels supported by the outer well in the $\mathrm{HCl}^{-}$curve (cf. Fig. 2).

The results computed with the NWE and BO-NWE models are compared with the experiment in Fig. 4. For the 0 $\rightarrow 1$ cross section, we show the experimental values of Schafer and Allan [34] from threshold to $0.5 \mathrm{eV}$; for collision energies above $0.5 \mathrm{eV}$, we use the more recent highresolution data of Allan et al. [19]. The $0 \rightarrow 2$ data is taken from Schafer and Allan and does not have the resolution needed to display the outer-well structures. To facilitate the comparison with theory, the numerical results were convoluted with a Gaussian of $20-\mathrm{meV}$ width. The experimental results were all reported in arbitrary units and were hence
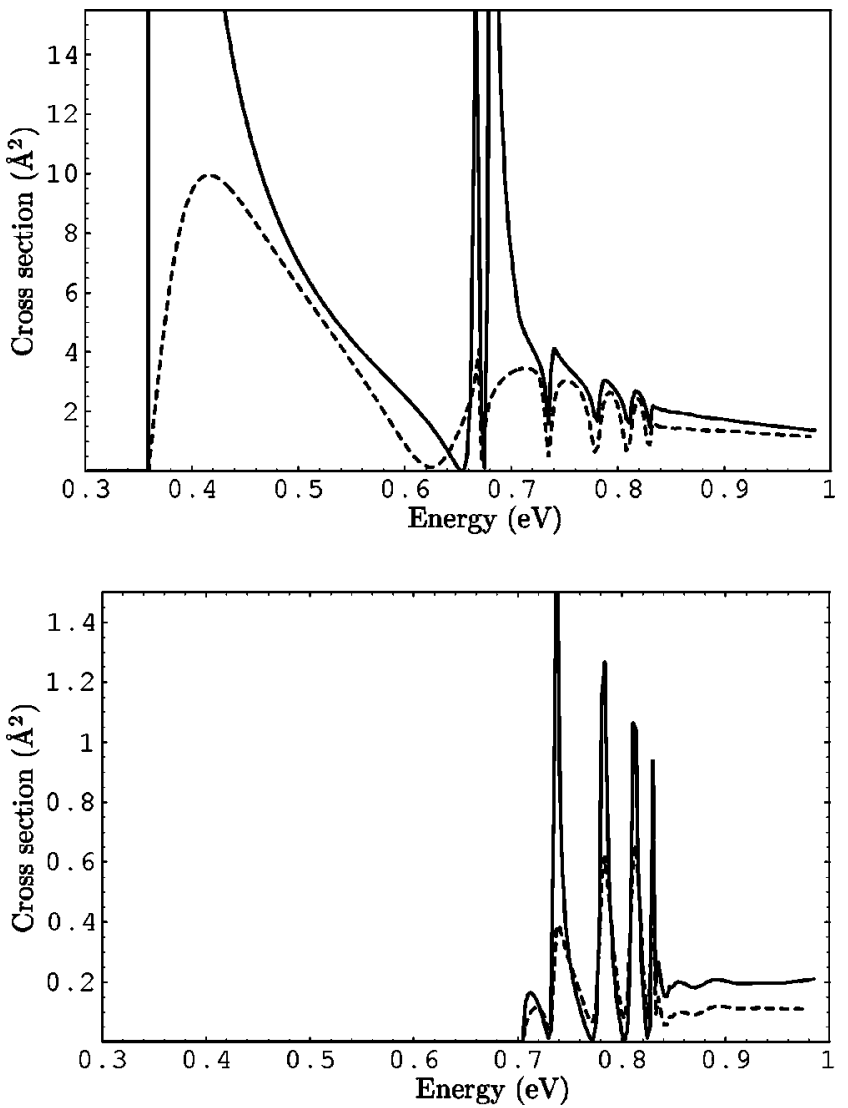

FIG. 3. Vibrational excitation cross sections for $\mathrm{HCl}$ computed with NWE and BO-NWE models. Solid curves, NWE; and dashed curves, BO-NWE. Top panel, $0 \rightarrow 1$ cross section; and bottom panel, $0 \rightarrow 2$ cross section.

normalized to the theoretical values. As previously mentioned, the calculations are extremely sensitive to details of the anion potential curve in the vicinity of its crossing with the neutral, and the ab initio data from which our models were constructed leaves some uncertainty about this region. Nevertheless, it is clear that the model calculations capture the essential features of the observed excitation cross sections.

\section{DISCUSSION}

We have formulated a model for low-energy electronmolecule scattering that can be used in situations where the collision dynamics is effected by the presence of a virtual state. The formulation is based on a zero-range model that approximates the electron-molecule interaction by a matching condition that is independent of energy but changes with target nuclear geometry.

The zero-range model was originally presented as an infinite set of linear equations with parameters that were adjusted to fit experimental observations. Our reformulation of the zero-range model has achieved two principal goals. The first is to obviate the need for a semiempirical determination of the model parameters by deriving an approximation to the complete anion potential curve using properties of electrondipole scattering. The advantage of such an approach is that 

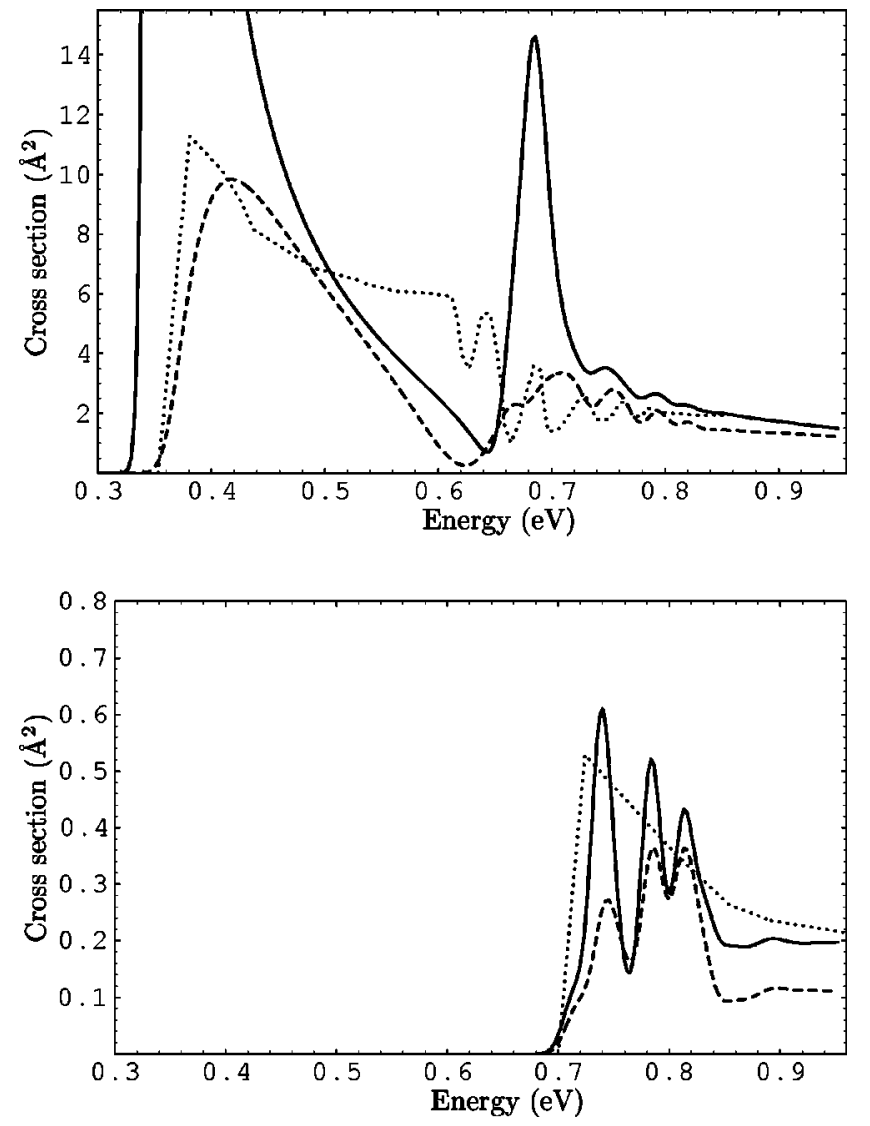

FIG. 4. Vibrational excitation cross sections for $\mathrm{HCl}$. Comparison of NWE and BO-NWE model results with experiment. Solid curves, NWE; dashed curves, BO-NWE; and dotted curves, experimental results (see text for explanation). Top panel, $0 \rightarrow 1$ cross section; and bottom panel, $0 \rightarrow 2$ cross section.

ab initio input can be provided by standard quantum chemistry tools since we only require information on neutral and anion potential curves for configurations where they are electronically bound. We also need the dipole moment of the neutral target as a function of nuclear geometry. In particular, fixed-nuclei electron-molecule scattering calculations are not required to determine the model parameters.
The second feature of the reformulated zero-range model is that the infinite system of linear equations can be recast in the form of an inhomogeneous differential equation or nuclear wave equation. This obviates the need for an expansion in target vibrational states, which can diverge at energies above the threshold for dissociative attachment. More importantly, it provides a viable path to extending the treatment to polyatomic targets.

To illustrate the method, we have revisited the problem of vibrational excitation of $\mathrm{HCl}$ by electron impact and showed, for the first time, that a simple zero-range model predicts the oscillating structures in the cross sections. These oscillations originate from the shallow outer well in the $\mathrm{HCl}^{-}$potential curve and have been predicted by the projection operator theory and confirmed experimentally. While our calculations reproduce essential features of the observed excitation cross sections, the correspondence with experimental data is not perfect. This is caused by uncertainties in the available $a b$ initio data we used as input and the inability to treat the $p$-wave shape resonance in our model, which is known to play an important role at higher energies. Indeed, a disadvantage of the current formulation is its limitation to low-energy $s$-wave scattering. There are many interesting problems in electron-molecule scattering where the cross sections display both low-energy virtual state effects and the effects of shape resonances at higher energies and the current approach is not able to deal with these situations in a unified way.

The present formulation of the zero-range model can be extended to small polyatomic targets with modest computational effort and calculations on the $e^{-}-\mathrm{CO}_{2}$ system are currently underway.

\section{ACKNOWLEDGMENTS}

This work was performed under the auspices of the U.S. Department of Energy by the University of California Lawrence Berkeley Laboratory under Contract No. DEAC03-76SF00098. The work was supported by the U.S. DOE Office of Basic Energy Science, Division of Chemical Sciences.
[1] K. Rohr and F. Linder, J. Phys. B 9, 2521 (1976).

[2] L. Dubé and A. Herzenberg, Phys. Rev. Lett. 38, 820 (1977).

[3] J.P. Gauyacq and A. Herzenberg, Phys. Rev. A 25, 2959 (1982)

[4] W. Domcke and L.A. Cederbaum, J. Phys. B 14, 149 (1981).

[5] T.F. O’Malley, Phys. Rev. 150, 14 (1966).

[6] B. Schneider, M.L. Dourneuf, and P. Burke, J. Phys. B 12, L365 (1979).

[7] L.A. Morgan and P.G. Burke, J. Phys. B 21, 2091 (1988).

[8] L.A. Morgan, P.G. Burke, and C.J. Gillan, J. Phys. B 23, 99 (1990).

[9] R. Fandreyer, P.G. Burke, L.A. Morgan, and C.J. Gillan, J. Phys. B 26, 3625 (1993).

[10] M. McCartney, P.G. Burke, L.A. Morgan, and C.J. Gillan, J.
Phys. B 23, L415 (1990).

[11] R. Fandreyer and P.G. Burke, J. Phys. B 29, 339 (1996).

[12] R.G. Newton, Scattering Theory of Particles and Waves, 2nd ed. (Springer-Verlag, New York, 1982).

[13] A. Herzenberg, J. Phys. B 17, 4213 (1984).

[14] R.S.M. Pont, P.M. Potvliege, and P.H.G. Smith, Phys. Rev. A 46, 555 (1992).

[15] W. Vanroose, P. Van Leuven, F. Arickx, and J. Broeckhove, J. Phys. A 30, 5543 (1997).

[16] N.J. Kylstra and C.J. Joachain, Phys. Rev. A 57, 412 (1998).

[17] W. Vanroose, Phys. Rev. A 64, 062708 (2001).

[18] W. Domcke, J. Phys. B 14, 4889 (1981).

[19] M. Allan, M. Cizek, J. Horacek, and W. Domcke, J. Phys. B 33, L209 (2000). 
[20] M. Cizek, J. Horacek, A.-C. Sergenton, D.B. Popovic, M. Allan, W. Domcke, T. Leininger, and F.X. Gadea, Phys. Rev. A 63, 062710 (2001).

[21] D.T. Birtwistle and A. Herzenberg, J. Phys. B 4, 53 (1971).

[22] T.N. Rescigno, W.A. Isaacs, A.E. Orel, H.-D. Meyer, and C.W. McCurdy, Phys. Rev. A 65, 032716 (2002).

[23] C.W. McCurdy, W.A. Isaacs, H.-D. Meyer, and T.N. Rescigno, Phys. Rev. A 67, 042708 (2003).

[24] M. Allan, J. Phys. B 35, L387 (2002).

[25] M. Allan, J. Phys. B 36, 2489 (2003).

[26] J.-M. Lévy-Leblond, Phys. Rev. 153, 1 (1967).

[27] A. Herzenberg and B.C. Saha, J. Phys. B 16, 591 (1983).

[28] J.-P. Gauyacq, Dynamics of Negative Ions (World Scientific,
Singapore, 1987).

[29] P. Astrand and G. Karlstrom, Chem. Phys. Lett. 175, 624 (1990).

[30] M. Cizek, J. Horacek, and W. Domcke, Phys. Rev. A 60, 2873 (1999).

[31] S.V. ONeil, P. Rosmus, D.W. Norcross, and H.-J. Werner, J. Chem. Phys. 85, 7232 (1986).

[32] D.E. Manolopoulos and R.E. Wyatt, Chem. Phys. Lett. 152, 23 (1988).

[33] T.N. Rescigno and C.W. McCurdy, Phys. Rev. A 62, 032706 (2000).

[34] O. Schafer and M. Allan, J. Phys. B 24, 3069 (1991). 